\title{
Rezension „Smart City konkret - Eine Zukunftswerkstatt in Deutschland zwischen Idee und Praxis"
}

\author{
Edy Portmann
}

Eingegangen: 26. Mai 2015 / Angenommen: 1. Juni 2015 / Online publiziert: 17. Juni 2015

(C) Springer Fachmedien Wiesbaden 2015

Hatzelhoffer, Lena; Humboldt, Kathrin; Lobeck, Michael und Wiegandt, Claus-Christian:

Smart City konkret - Eine Zukunftswerkstatt in Deutschland zwischen Idee und Praxis

ISBN 978-3-86859-161-3, Jovis Verlag GmbH, Berlin 2012, 272 S., € 39.80

Im erfrischend positiv geschriebenen Fachbuch ,Smart City konkret - Eine Zukunftswerkstatt in Deutschland zwischen Idee und Praxis“" werden die Erkenntnisse vom Projekt „T-City“ als Endbericht aufbereitet. Als „T-City“ wird das Smart City Projekt der Stadt Friedrichshafen bezeichnet, welche in einer Public Private Partnership mit der Deutschen Telekom (und weiteren Vertretern aus der Privatwirtschaft) von 2006 bis 2012 den ambitionierten Versuch wagte, die erste ,echte“ Smart City Deutschlands zu werden. In diesem Bericht heben die Autoren - Mitarbeiter der Arbeitsgruppe Stadt- und Regionalforschung des Geographischen Instituts der Universität Bonn - die konkrete Realisierung einer Smart City als große Besonderheit des ,T-City“ Projekts gegenüber anderer Smart City Vorhaben heraus.

Für ihr Buch - welches wie eine ausgedehnte Hochglanzbroschüre daherkommt - wählten die Autoren eine originelle, am Lebenszyklus von Informations- und Kommunikationstechnologie (IKT) orientierte Gliederung: Im Einleitungskapitel

„Enter“ beschreiben sie überblicksartig, dass Smart City Programme zuerst entwickelt werden müssen (Kap. 2 „Entwickeln“), bevor sie in der Stadt installiert werden können (Kap. 3 „Installieren“). Diese Programme werden dann von verschiedenen Akteuren geöffnet und genutzt (Kap. 4 ,Öffnen“). Dabei weisen die Autoren darauf hin, dass immer die Gefahr eines Programmabsturzes besteht (Kap. 5 „Absturz vermeiden"). Zum Abschluss gehen die Autoren noch auf die im Verlaufe der Zeit

E. Portmann $(\bowtie)$

Bern, Schweiz

E-Mail: edy.portmann@iwi.unibe.ch 
erforderlichen Updates einer Smart City ein (Kap. 6 „Update“). Diese Gliederung überzeugt mich, da sie den praxisorientierten Kern gesammelter Erfahrungen einer Smart City unterstreicht.

Im Kapitel „Entwickeln“ werden Smart Cities begrifflich gefasst sowie das Projekt ,T-City“ in einem - (leider) sehr Deutschlandlastigen - Framework betrachtet. Im Jahr 2006 rief die Deutsche Telekom zu einem richtungsweisenden Wettbewerb deutscher Städte auf. Sie sollten nach Ideen suchen, wie mit IKT ,ganzheitlich urbane Lebensbereiche integriert und miteinander vernetzt werden können" (ihre Arbeitsdefinition). Als Fan gestaltungsorientierter Zusammenarbeit empfinde ich diesen Wettbewerb als mutiges, aber gelungenes Unterfangen. Im Kapitel „Installieren“ gehen die Autoren auf einzelne IKT-Projekte (z. B. Bildung, Energie und/oder Gesundheit) ein, welche in der Stadt am Bodensee realisiert wurden - oder hätten realisiert werden sollen. Leider kommt hier die technische Sichtweise - die den einen oder andere durch den Deutschen Telekomriesen angelockte Leser erwarten könnte - neben der soziologischen Sichtweise zu kurz.

Das Kapitel „Öffnen“ beschäftigt sich neben der IKT-Nutzung (und ihrer Auswirkung auf das Stadtleben) auch damit, wie die „T-City“ wahrgenommen und erlebt wird. Dazu fokussieren sich die Autoren auf die Bürger, beleuchten deren Probleme anhand einzelner (ausgewählter) ,T-City“ Projekte (wie eben Bildung, Energie und/ oder Gesundheit). Hier setzen die Autoren - wieder aus soziologischer Perspektive, aber dieses Mal passend(er) - ihren Fokus zu Recht auf die Bürger von Friedrichshafen, welche in ihrer Smart City leben. Das Kapitel „Absturz vermeiden“ adressiert den Spagat, welchen die Beteiligten der „T-City“ machen müssen, um Projektfiaskos zu vermeiden bspw. um ihre Daten zu sichern und schützen. Meine Prophezeiung ist, dass sich die Sorgen um den Schutz und um die Sicherheit der Daten auch als Knackpunkt für die Realisierung anderer angehender Smart Citys herausstellen wird. Das abschließende Kapitel „Update“ nimmt letztendlich nochmals die wesentlichen Punkte auf. Ein Highlight sind die Empfehlungen (sog. Best Practices) für andere - ,emergente“ - Smart Cities: Ziel- und Rollenerwartungen klären, (offene) Strukturen (bspw. in der Kommunikation und Kultur), regelmäßige Reflexionen und (externe) Evaluationen etc.

Auf alle sechs Kapitel verteilt kommen verschiedene Akteure der „T-City“ (z. B. Bürgermeister, Forscher, Projektleiter und Zukünftler) in Kurzberichten zu Wort. (Zur Info: Als Zukünftler werden all die Glücklichen bezeichnet, welche als Gewinner einer Ausschreibung direkte Erfahrungen mit „zukünftiger“ Technik sammeln konnten). Die einzelnen - meines Erachtens teilweise zu Akademielastigen - Stellungnahmen kommen mal gewitzt, manchmal aber auch - leider - ein wenig (zu) fade daher.

Fazit: Das Fachbuch „Smart City konkret - Eine Zukunftswerkstatt in Deutschland zwischen Idee und Praxis" sei all jenen Smart City Interessierten, wie bspw. Bürgermeistern, Smart City Projektleitern und -teilnehmern, empfohlen, welche sich über bereits realisierte Projekte und dabei gemachten Erfahrungen informieren wollen. Die aufwändige und bilderreiche Gestaltung des Buches (mit vielen als QR-Code eingefügten Links zu weiterführenden Quellen) kann zudem noch den einen oder anderen unentschlossenen Käufer ansprechen. Ich empfehle es, mit dem Grundgedanken aus gemachten Erfahrungen zu lernen, auf jeden Fall weiter. 